EPJ Web of Conferences 49, 13002 (2013)

DOI: $10.1051 /$ epjconf/20134913002

(C) Owned by the authors, published by EDP Sciences, 2013

\title{
Rare beauty and charm decays with the CMS experiment
}

\author{
Kai-Feng Chen ${ }^{1, a}$ \\ ${ }^{1}$ National Taiwan University, Taipei, Taiwan
}

\begin{abstract}
Rare beauty meson decays $\mathrm{B}_{\mathrm{s}}^{0} \rightarrow \mu^{+} \mu^{-}, \mathrm{B}^{0} \rightarrow \mu^{+} \mu^{-}$, and charm meson decays $\mathrm{D}^{0} \rightarrow \mu^{+} \mu^{-}$are searched in pp collisions at $\sqrt{s}=7 \mathrm{TeV}$. A data sample corresponding to an integrated luminosity of $5 \mathrm{fb}^{-1}$ collected by the CMS experiment is used for the $\mathrm{B} \rightarrow \mu^{+} \mu^{-}$study, while the data sample used for $\mathrm{D}^{0} \rightarrow \mu^{+} \mu^{-}$ search is $90 \mathrm{pb}^{-1}$. The number of events observed after applying the full selection criteria is consistent with the expectations from the sum of background and standard model signal. The resulting upper limits on the branching fractions are $\mathcal{B}\left(\mathrm{B}_{\mathrm{s}}^{0} \rightarrow \mu^{+} \mu^{-}\right)<7.7 \times 10^{-9}, \mathcal{B}\left(\mathrm{B}^{0} \rightarrow \mu^{+} \mu^{-}\right)<1.8 \times 10^{-9}$ at $95 \%$ confidence level, and $\mathcal{B}\left(\mathrm{D}^{0} \rightarrow \mu^{+} \mu^{-}\right)<5.4 \times 10^{-7}$ at $90 \%$ confidence level.
\end{abstract}

\section{Search for rare $\mathrm{B}_{\mathrm{s}}^{0} \rightarrow \mu^{+} \mu^{-}$and $\mathrm{B}^{0} \rightarrow \mu^{+} \mu^{-}$ decays}

The decays $\mathrm{B}_{\mathrm{s}}^{0} \rightarrow \mu^{+} \mu^{-}$and $\mathrm{B}^{0} \rightarrow \mu^{+} \mu^{-}$are highly suppressed in the standard model of particle physics. As shown in Fig. 1, these processes only proceed through the flavor-changing neutral currents, which are forbidden at leading order diagrams; an internal quark annihilation within the $\mathrm{B}$ meson is also required and further reduces the decay rate. Further suppression based on helicity is given by a factor of $m_{\mu}^{2} / m_{\mathrm{B}}^{2}$, where $m_{\mu}\left(m_{\mathrm{B}}\right)$ is the mass of the muon (B meson). The predicted branching fractions of these two decays are given by $\mathcal{B}\left(\mathrm{B}_{\mathrm{s}}^{0} \rightarrow \mu^{+} \mu^{-}\right)=$ $(3.2 \pm 0.2) \times 10^{-9} \mathcal{B}\left(\mathrm{B}^{0} \rightarrow \mu^{+} \mu^{-}\right)=(1.0 \pm 0.1) \times 10^{-10}[1]$, and are particularly reliable. Several standard model extensions, such as a two-Higgs doublet model [2] or a specific model with leptoquarks [3], may introduce enhancements to the branching fractions. Some other new physics models, the decay rates can also be reduced [4]. These decays are an excellent probe to the new physics; any difference in standard model branching fraction could provide a smoking gun signal.

Simultaneous searches for $\mathrm{B}_{\mathrm{s}}^{0} \rightarrow \mu^{+} \mu^{-}$and $\mathrm{B}^{0} \rightarrow \mu^{+} \mu^{-}$ decays using data collected by the CMS experiment in pp collisions at $\sqrt{s}=7 \mathrm{TeV}$ are presented in Ref. ??. A dataset corresponding to an integrated luminosity of $5 \mathrm{fb}^{-1}$ is used. The CMS detector is a general-purpose detector, a detailed description can be found in Ref. [6]. The central feature of the detector is a superconducting solenoid that provides an axial magnetic field of $3.8 \mathrm{~T}$. Charged particle trajectories are measured within the field volume by pixel and silicon strip tracker. The calorimeter enclosing the tracker includes a lead tungstate crystal electromagnetic calorimeters (ECAL), which is composed of a barrel part and two endcaps, a lead and silicon preshower detector in front of the ECAL endcaps, and a brass/scintillator

a e-mail: Kai-Feng.Chen@cern.ch
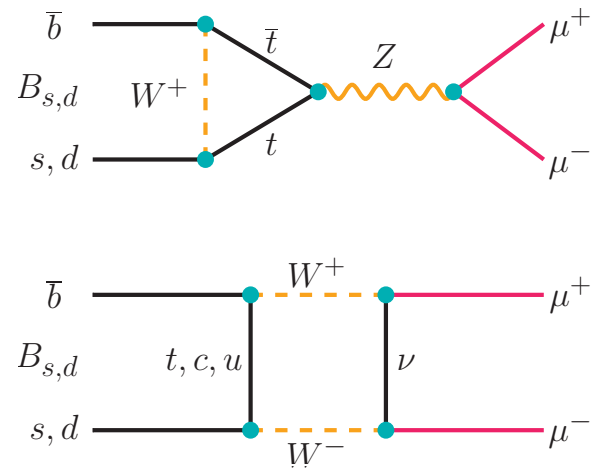

Figure 1. Representative Feynman diagrams for $\mathrm{B}_{\mathrm{s}}^{0} \rightarrow \mu^{+} \mu^{-}$and $\mathrm{B}^{0} \rightarrow \mu^{+} \mu^{-}$decays.

hadron calorimeter (HCAL) that together provide an energy measurement for electrons, photons, and hadronic jets. Muons are identified and measured in gas-ionization detectors embedded in the steel return yoke outside the solenoid. The detector is nearly hermetic, allowing accurate energy balance measurements in the plane transverse to the beam direction. The direction of particles measured inside the CMS detector is described using the azimuthal angle $(\phi)$ and the pseudorapidity $(\eta)$, which is defined as $\eta \equiv-\ln [\tan \theta / 2]$, where $\theta$ is the polar angle relative to the counterclockwise proton beam direction, as measured from the nominal interaction vertex.

\subsection{Reconstruction and selection}

Muon candidates are reconstructed by combining tracks found in the silicon tracker and the muon detector. The muon candidates must have at least two track segments in the muon stations, and have more than ten hits in the silicon tracker, while at least one must be in the pixel detector. The track-fit quality ( $\chi^{2}$ per degree of freedom) for the 
combined track must be less than 10 . The impact parameter in the transverse plane with respect to the beam-spot must be smaller than $0.2 \mathrm{~cm}$. The muon efficiency is determined with a tag-and-probe method with $J / \psi \rightarrow \mu^{+} \mu^{-}$ events, which has been applied to both data and simulated samples. For the signal events, the average efficiency is estimated to be $71 \%(85 \%)$ in the barrel (endcap) channel based on the simulations.

Reconstruction of $\mathrm{B} \rightarrow \mu^{+} \mu^{-}$candidates requires two oppositely-charged muons that originate from a common vertex, and the invariant mass of the pair must be within the range of $4.9<m_{\mu \mu}<5.9 \mathrm{GeV} / c^{2}$. The dimuon mass resolution, which is a critical factor for separating $\mathrm{B}_{\mathrm{s}}^{0}$ and $\mathrm{B}^{0}$ decays, highly depends on the pseudorapidity $\eta$ of the reconstructed particles. The background level also depends on the direction of the reconstructed B candidate. The analysis is performed in two subchannels, "barrel" (where both muons have $|\eta|<1.4$ ) and "endcap" (where at least one muon has $|\eta|>1.4$ ).

The primary vertex is chosen to be the one which has minimal separation from the $\mathrm{z}$ intercept of the extrapolated $\mathrm{B}$ candidate track, along the $\mathrm{z}$ axis; systematic effects due to pileup are largely removed this primary vertex selection. The $3 \mathrm{D}$ impact parameter of the B candidate $\left(\delta_{3 D}\right)$, the uncertainty $\sigma\left(\delta_{3 D}\right)$, and its significance $\delta_{3 D} / \sigma\left(\delta_{3 D}\right.$ are measured with respect to this primary vertex.

Three variables are used for adopting the isolation information of the B candidates: (i) the isolation $I=$ $p_{T}(\mathrm{~B}) /\left(p_{T}(\mathrm{~B})+\sum_{\text {trk }} p_{T}\right)$ is calculated from the transverse momentum of the $\mathrm{B}$ candidate $p_{T}(\mathrm{~B})$ and the transverse momenta of other charged tracks within a cone of $\Delta R<$ 0.7; (ii) the number of tracks $N_{\text {trk }}^{\text {close }}$ with the distance of closest approach $<0.03 \mathrm{~cm}$, with respect to the $\mathrm{B}$ vertex; (iii) the minimum distance of closest approach between tracks and the $\mathrm{B}$ vertex $\left(d_{\mathrm{ca}}^{0}\right)$. These variables help to reject partly reconstructed $\mathrm{B}$ decays with other tracks, in addition to the two muons, associated with the $\mathrm{B}$ vertex.

In addition to the isolation related variables, the 3D pointing angle $\alpha$, the 3D flight length significance $\ell_{3 D} / \sigma\left(\ell_{3 D}\right)$, and the $\chi^{2} /$ dof of the vertex fit, are also examined.

The requirements on the variables discussed above, together with transverse momenta of the $\mathrm{B}$ candidate and two daughter muons, are optimized to obtain the best expected upper limit using simulated signal events and data background events. The data background events are defined as B candidates with a dimuon mass in the sidebands covering the range $4.9<m_{\mu \mu}<5.9 \mathrm{GeV} / c^{2}$, excluding the signal windows of $5.20<m_{\mu \mu}<5.45 \mathrm{GeV} / c^{2}$. The optimized selection criteria, which are used to determine the final results, are summarized in Table 1.

After the selection requirements, simulated events are used to determine the efficiencies for the signal samples. The simulated events are generated with Рүтніa 6.424 (TunE Z2) [7] and the B mesons are decayed via EvTGEN [8].
Table 1. Selection criteria for the events in the barrel and endcap channel.

\begin{tabular}{l|c|c|l}
\hline Variable & Barrel & Endcap & units \\
\hline$p_{T \mu, 1}>$ & 4.5 & 4.5 & $\mathrm{GeV}$ \\
$p_{T \mu, 2}>$ & 4.0 & 4.2 & $\mathrm{GeV}$ \\
$p_{T \mathrm{~B}}>$ & 6.5 & 8.5 & $\mathrm{GeV}$ \\
$\delta_{3 D}<$ & 0.008 & 0.008 & $\mathrm{~cm}$ \\
$\delta_{3 D} / \sigma\left(\delta_{3 D}\right)<$ & 2.000 & 2.000 & \\
$\alpha<$ & 0.050 & 0.030 & $\mathrm{rad}$. \\
$\chi^{2} /$ dof $<$ & 2.2 & 1.8 & \\
$\ell_{3 D} / \sigma\left(\ell_{3 D}\right)>$ & 13.0 & 15.0 & \\
$I>$ & 0.80 & 0.80 & \\
$d_{\text {ca }}^{0}>$ & 0.015 & 0.015 & $\mathrm{~cm}$ \\
$N_{\text {trk }}^{\text {close }}<$ & 2 & 2 & \\
\hline
\end{tabular}

\subsection{Results}

A counting experiment in dimuon mass regions is carried out in a target signal region of $5.20<m_{\mu \mu}<5.45 \mathrm{GeV} / c^{2}$. Combinatorial backgrounds are evaluated from the data events outside of signal region. The backgrounds due to rare B decays, where one or two hadrons are misidentified as muons, are estimated with simulated samples. These decays include $\mathrm{B} \rightarrow h^{+} \mu^{-} v$ and $\mathrm{B} \rightarrow h^{+} h^{-}$, where $h^{ \pm}$denotes a charged pion, kaon or proton and the mother particle $\mathrm{B}$ could be $\mathrm{B}^{0}, \mathrm{~B}_{s}$, or $\Lambda_{b}$.

A normalization channel $\mathrm{B}^{+} \rightarrow J / \psi \mathrm{K}^{+}$is introduced to remove the uncertainties related to the $\mathrm{b}$-quark production cross section and to the integrated luminosity. The reconstruction of $\mathrm{B}^{+} \rightarrow J / \psi \mathrm{K}^{+}$events is very similar to the reconstruction of $\mathrm{B} \rightarrow \mu^{+} \mu^{-}$events. Candidates with two oppositely-charged muons associating with a common vertex are required to have an invariant mass within the range of 3.0 to $3.2 \mathrm{GeV} / c^{2}$. These candidates are combined with another track which is assumed to be a kaon. All requirements introduced for $\mathrm{B} \rightarrow \mu^{+} \mu^{-}$events are also applied.

Branching fractions (and the associated upper limits) are measured in the barrel and endcap channels separately using the following formula

$$
\mathcal{B}\left(\mathrm{B} \rightarrow \mu^{+} \mu^{-}\right)=\frac{N_{\mathrm{S}}}{N_{\mathrm{obs}}^{\mathrm{B}^{+}}} \frac{f_{u}}{f_{s}} \frac{\varepsilon_{\mathrm{tot}}^{\mathrm{B}^{+}}}{\varepsilon_{\mathrm{tot}}} \mathcal{B}\left(\mathrm{B}^{+}\right),
$$

where $\varepsilon_{\text {tot }}\left(\varepsilon_{\text {tot }}^{\mathrm{B}^{+}}\right)$is the total signal (normalization channel) efficiency, and $N_{\mathrm{S}}\left(N_{\mathrm{obs}}^{\mathrm{B}^{+}}\right)$is the yield of $\mathrm{B} \rightarrow \mu^{+} \mu^{-}$ $\left(\mathrm{B}^{+} \rightarrow J / \psi \mathrm{K}^{+}\right)$decays. Branching fraction of the normalization channel $\mathcal{B}\left(\mathrm{B}^{+}\right)$is given by PDG [9], and the LHCb measured value of $f_{s} / f_{u}=0.267 \pm 0.021$ [10] is used, which is the ratio of the $\mathrm{B}^{+}$and $\mathrm{B}_{\mathrm{s}}$ production cross sections.

The yields of $\mathrm{B}^{+} \rightarrow J / \psi \mathrm{K}^{+}$decays are determined to be $(82.7 \pm 4.2) \times 10^{3}$ and $(23.8 \pm 1.2) \times 10^{3}$ in the barrel and endcap, respectively, with fits to the invariant mass distributions shown in Fig. 2. The total efficiencies $\varepsilon_{\text {tot }}^{\mathrm{B}^{+}}$ are estimated to be $(11.0 \pm 0.9) \times 10^{-4}$ for the barrel and $(3.2 \pm 0.4) \times 10^{-4}$ for the endcap channel.

Expected yields of signal $N_{\text {signal }}^{\exp }$ (assuming the standard model branching fractions) are summarized in Tab. 2 . 

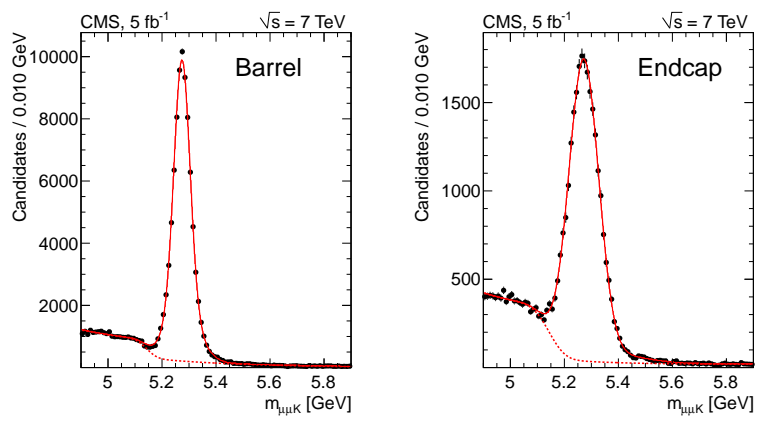

Figure 2. Invariant-mass distributions of the normalization channel $\left(\mathrm{B}^{+} \rightarrow J / \psi \mathrm{K}^{+}\right)$in the barrel (left) and endcap (right). The solid and dashed lines show the fits to the data and background, respectively.

Expected yields of background from rare B decays, $N_{\text {peak }}^{\text {exp }}$, are also shown in the same table. Those yields are all scaled according to the normalization channel, including the performance of muon detection efficiency and misidentification rates determined from data. The expected numbers of combinatorial background events, $N_{\text {comb }}^{\text {exp }}$, are evaluated by an interpolation from the events in the sideband regions assuming a flat background model, while the expected rare semileptonic backgrounds are subtracted.

The measured dimuon invariant mass distributions around the B meson mass are shown in Fig. 3. The observed yields, as summarized in Tab. 2 as well, are consistent with the expectations for standard model signals plus background sources. Upper limits on the $\mathrm{B}_{\mathrm{s}} \rightarrow \mu^{+} \mu^{-}$ and $\mathrm{B}^{0} \rightarrow \mu^{+} \mu^{-}$branching fractions are determined using the $\mathrm{CL}_{\mathrm{s}}$ method $[11,12]$. The upper limits are evaluated to be $\mathcal{B}\left(\mathrm{B}_{\mathrm{s}} \rightarrow \mu^{+} \mu^{-}\right)<7.7 \times 10^{-9}\left(6.4 \times 10^{-9}\right)$ and $\mathcal{B}\left(\mathrm{B}^{0} \rightarrow \mu^{+} \mu^{-}\right)<1.8 \times 10^{-9}\left(1.4 \times 10^{-9}\right)$ at $95 \%(90 \%)$ confidence level. Including possible cross-feed events between the $\mathrm{B}_{\mathrm{s}}$ and $\mathrm{B}^{0}$ decays, the background-only $p$ values are calculated to be $0.11(1.2 \sigma)$ and $0.24(0.7 \sigma)$ for $\mathrm{B}_{\mathrm{s}} \rightarrow \mu^{+} \mu^{-}$and $\mathrm{B}^{0} \rightarrow \mu^{+} \mu^{-}$, respectively.

In summary, the searches for the rare $\mathrm{B}$ decays $\mathrm{B}_{\mathrm{s}} \rightarrow$ $\mu^{+} \mu^{-}$and $\mathrm{B}^{0} \rightarrow \mu^{+} \mu^{-}$have been carried out in pp collisions at $\sqrt{s}=7 \mathrm{TeV}$. A data sample corresponding to an integrated luminosity of $5 \mathrm{fb}^{-1}$ has been used. The observed number of events is consistent with background plus signals based on the standard model predicted branching fractions, and no significant excess found. The upper limits on the branching fractions have been evaluated and can be used to improve the bounds on several extensions of the standard model.

\section{Search for rare $\mathrm{D}^{0} \rightarrow \mu^{+} \mu^{-}$decays}

The rare charm decay $\mathrm{D}^{0} \rightarrow \mu^{+} \mu^{-}$shares the same diagrams of $\mathrm{B}^{0} \rightarrow \mu^{+} \mu^{-}$; the flavor-changing neutral current decays are rare and only proceed via the process with an internal loop. The predicted branching fraction [13] is approximately $10^{-18}$ from short range processes and can
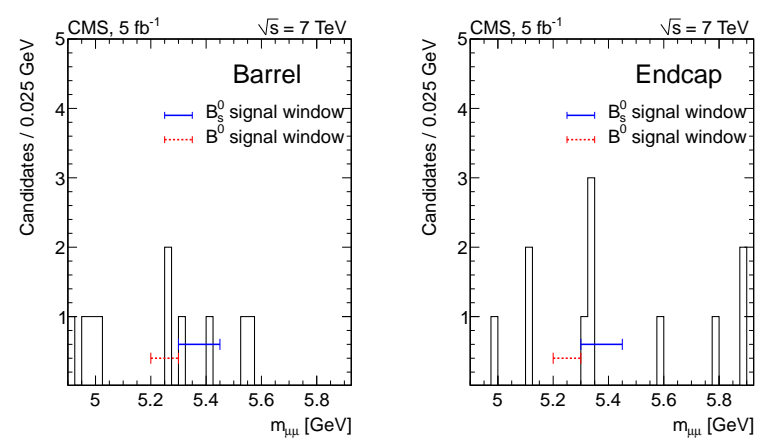

Figure 3. Dimuon invariant-mass distributions for the barrel channel (left) and endcap channel (right). The horizontal lines show the signal regions for $\mathrm{B}_{\mathrm{s}}$ and $\mathrm{B}^{0}$ modes.

be enlarged to $10^{-13}$ with long range contributions. Any observation of these modes at rates exceeding the prediction of the standard model could hint the existence of new physics.

A search for $\mathrm{D}^{0} \rightarrow \mu^{+} \mu^{-}$is performed using a dataset of pp collisions at $\sqrt{s}=7 \mathrm{TeV}$, corresponding to an integrated luminosity of $90 \mathrm{pb}^{-1}$, collected by the CMS detector. The CMS detector has been introduced in the previous section already. The analysis is carried out by measuring the productions of $\mathrm{D}^{*+} \rightarrow \mathrm{D}^{0} \pi^{+}, \mathrm{D}^{0} \rightarrow \mu^{+} \mu^{-}$, respect to the normalization channel $\mathrm{D}^{*+} \rightarrow \mathrm{D}^{0} \pi^{+}, \mathrm{D}^{0} \rightarrow \mathrm{K}^{-} \mu^{+} v$. Most of the systematics can be cancelled, however, the challenge and restriction of this analysis is to reconstruct the $\mathrm{D}^{0} \rightarrow \mathrm{K}^{-} \mu^{+} v$ events. The trigger efficiency is much smaller due to the higher threshold at trigger level, and also restricts the amount of data that can be included in the study.

\subsection{Reconstruction and selection}

The events with a muon having a $p_{T}$ greater than a certain trigger threshold, which changes with running conditions, are selected for the analysis. The dataset collected in 2010 has six different trigger thresholds, varies from 3 to $15 \mathrm{GeV} / c$ on the muon $p_{T}$. The first $54 \mathrm{pb}^{-1}$ of data collected in 2011 have a $p_{T}$ threshold of $15 \mathrm{GeV} / c$. The later data sets are not included in this analysis due to the low reconstruction efficiency of the normalization decay $\mathrm{D}^{0} \rightarrow \mathrm{K}^{-} \mu^{+} v$.

The signal $\mathrm{D}^{0} \rightarrow \mu^{+} \mu^{-}$candidate is built with two oppositely charged muon candidates. Muon candidates are required to be reconstructed both in the silicon tracker and in the muon stations. The global fit of the muon track (in the tracker and muon system) must have a good quality: $\chi^{2}$ per degree of freedom less than 3 . The distance of closest approach between the primary vertex and the muon track should be with in $2 \mathrm{~mm}$ in the transverse plane. One of the two muons must fire the trigger, which implies the transverse momentum of this muon candidate much be above the trigger threshold. Another muon candidate must have a $p_{T}>3 \mathrm{GeV} / c$. These two muons must share a common vertex, with a minimum confidence level of $1 \%$ of the vertex finding fit. The angle between the $\mathrm{D}^{0}$ momentum 
Table 2. Signal selection efficiency $\left(\varepsilon_{\mathrm{tot}}\right)$, the predicted signal yields $\left(N_{\mathrm{exp}}^{\text {signal }}\right)$, the expected peaking and combinatorial background yields $\left(N_{\exp }^{\text {peak }}\right.$ and $\left.N_{\exp }^{\text {comb }}\right)$, the total number of expected events $\left(N_{\exp }^{\text {total }}\right)$, and the number of observed events $\left(N_{\text {obs }}\right)$ in the barrel and endcap channels. The uncertainties shown include both statistical and systematic sources.

\begin{tabular}{l|c|c|c|c}
\hline & $\mathrm{B}^{0} \rightarrow \mu^{+} \mu^{-}$Barrel & $\mathrm{B}_{\mathrm{s}} \rightarrow \mu^{+} \mu^{-}$Barrel & $\mathrm{B}^{0} \rightarrow \mu^{+} \mu^{-}$Endcap & $\mathrm{B}_{\mathrm{s}} \rightarrow \mu^{+} \mu^{-}$Endcap \\
$\varepsilon_{\text {tot }}(\%)$ & $0.29 \pm 0.02$ & $0.29 \pm 0.02$ & $0.16 \pm 0.02$ & $0.16 \pm 0.02$ \\
\hline$N_{\text {exp }}^{\text {sinal }}$ & $0.24 \pm 0.02$ & $2.70 \pm 0.41$ & $0.10 \pm 0.01$ & $1.23 \pm 0.18$ \\
$N_{\text {exp }}^{\text {peak }}$ & $0.33 \pm 0.07$ & $0.18 \pm 0.06$ & $0.15 \pm 0.03$ & $0.08 \pm 0.02$ \\
$N_{\text {exp }}^{\text {comb }}$ & $0.40 \pm 0.34$ & $0.59 \pm 0.50$ & $0.76 \pm 0.35$ & $1.14 \pm 0.53$ \\
\hline$N_{\text {exp }}^{\text {total }}$ & $0.97 \pm 0.35$ & $3.47 \pm 0.65$ & $1.01 \pm 0.35$ & $2.45 \pm 0.56$ \\
\hline$N_{\text {obs }}$ & 2 & 2 & 0 & 4 \\
\hline
\end{tabular}

vector and the flight direction of the $\mathrm{D}^{0}$ candidate must satisfy a $\cos \alpha>0.99$ requirement. The $\mathrm{D}^{*+}$ candidate is combined by a $\mathrm{D}^{0}$ candidate and a track, which are originated from the same primary vertex. A requirement on the $\Delta M=M\left(\mu^{+} \mu^{-} \pi^{+}\right)-M\left(\mu^{+} \mu^{-}\right)<180 \mathrm{MeV} / c^{2}$ is applied.

Reconstruction of $\mathrm{D}^{0} \rightarrow \mathrm{K}^{-} \mu^{+} v$ starts with combining a kaon candidate and an oppositely charged muon candidate. The muon candidate must satisfy the same requirements mentioned above. A secondary vertex is formed by the kaon and muon candidates and their vertex fit confidence level must be $>1 \%$. The technique developed by E691 experiment [14] is used to determine the neutrino momentum. Again the $\mathrm{D}^{*+}$ candidate is combined by a $\mathrm{D}^{0}$ candidate and a track with pion mass assumed. A requirement on the $\Delta M=M\left(\mathrm{~K}^{-} \mu^{+} v \pi^{+}\right)-M\left(\mathrm{~K}^{-} \mu^{+} v\right)<180$ $\mathrm{MeV} / \mathrm{c}^{2}$ is also applied. If there are multiple combinations exist, the combination with $\Delta M$ closest to the nominal PDG mass difference is selected. Significance of the separation between primary and secondary vertices is required to be greater than 3 .

Figure 4 shows the reconstructed normalization channel $\mathrm{D}^{*+} \rightarrow \mathrm{D}^{0}\left(\rightarrow \mathrm{K}^{-} \mu^{+} v\right) \pi^{+}$candidates and signal $\mathrm{D}^{*+} \rightarrow$ $\mathrm{D}^{0}\left(\rightarrow \mu^{+} \mu^{-}\right) \pi^{+}$events. With an additional requirement of $\left|\Delta M-\Delta M_{\mathrm{PDG}}\right|<3 \mathrm{MeV} / c^{2}$, the dimuon invariant mass distribution of the remaining events is shown in Fig. 5. The background shape in this limited mass region is assumed to be flat, and the background yield in the signal region is estimated from the yields in the sideband regions. There are 23 events observed in the signal region but the number of background events is also estimated to be 23 . Clearly there is no evidence of $\mathrm{D}^{0} \rightarrow \mu^{+} \mu^{-}$decays observed in data.

The limit of the $\mathrm{D}^{0} \rightarrow \mu^{+} \mu^{-}$decay branching fraction is evaluated with the following equation

$$
\mathcal{B}\left(\mathrm{D}^{0} \rightarrow \mu^{+} \mu^{-}\right) \leq \mathcal{B}\left(\mathrm{D}^{0} \rightarrow \mathrm{K}^{-} \mu^{+} v\right) \times \frac{N_{\mu \mu}}{N_{\mathrm{K} \mu \nu}} \times \frac{\varepsilon_{\mathrm{K} \mu v}}{\varepsilon_{\mu \mu}},
$$

where $N_{\mu \mu}$ is the upper limit on the $\mathrm{D}^{0} \rightarrow \mu^{+} \mu^{-}$signal yield, and $N_{\mathrm{K} \mu \nu}$ is the yield of normalization channel $\mathrm{D}^{0} \rightarrow$ $\mathrm{K}^{-} \mu^{+} v$. The overall efficiencies $\varepsilon_{\mathrm{K} \mu \nu}$ and $\varepsilon_{\mu \mu}$ include the effects of acceptance and reconstruction efficiencies and are extracted from simulated samples. The branching fraction of the normalization channel $\mathcal{B}\left(\mathrm{D}^{0} \rightarrow \mathrm{K}^{-} \mu^{+} v\right)$ is taken from the PDG.

In conclusion, the search of rare charm meson decay $\mathrm{D}^{0} \rightarrow \mu^{+} \mu^{-}$is performed at CMS experiment. No ex-
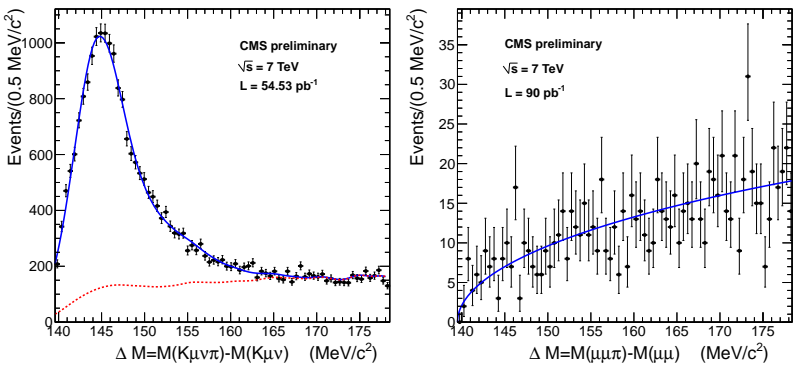

Figure 4. The $\Delta M$ distributions in the normalization channel $\mathrm{D}^{*+} \rightarrow \mathrm{D}^{0} \pi^{+}, \mathrm{D}^{0} \rightarrow \mathrm{K}^{-} \mu^{+} v$ (left), and in the signal channel $\mathrm{D}^{*+} \rightarrow \mathrm{D}^{0} \pi^{+}, \mathrm{D}^{0} \rightarrow \mu^{+} \mu^{-}$(right). In the left plot the background function is shown as a dashed curve.

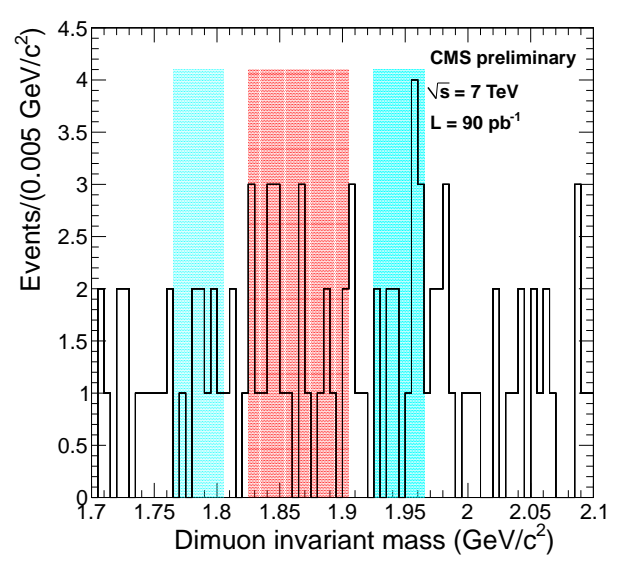

Figure 5. The dimuon invariant mass distribution for $\mathrm{D}^{0} \rightarrow \mu^{+} \mu^{-}$ candidates, after a requirement on the $\Delta M$. The red band shows the signal region, while the two blue bands show the sideband regions.

cess above the background seen in a dataset corresponding to an integrated luminosity of $90 \mathrm{pb}-1$. Based on the $\mathrm{CL}_{\mathrm{s}}$ method, the results from datasets with different trigger thresholds are combined. Several systematic sources, such as trigger efficiency, fit to the normalization channel, and the uncertainty on the PDG average value, have been studied and included in the limit calculations. The resulting limit is evaluated to be $\mathcal{B}\left(\mathrm{D}^{0} \rightarrow \mu^{+} \mu^{-}\right)<5.4 \times 10^{-7}$ at $90 \%$ confidence level. 


\section{References}

[1] A. J. Buras, Acta Phys. Polon. B 41, 2487 (2010) [arXiv:1012.1447 [hep-ph]].

[2] J. R. Ellis, K. A. Olive, Y. Santoso and V. C. Spanos, JHEP 0605, 063 (2006) [hep-ph/0603136].

[3] S. Davidson and S. Descotes-Genon, JHEP 1011, 073 (2010) [arXiv:1009.1998 [hep-ph]].

[4] J. R. Ellis, J. S. Lee and A. Pilaftsis, Phys. Rev. D 76, 115011 (2007) [arXiv:0708.2079 [hep-ph]].

[5] S. Chatrchyan et al. [CMS Collaboration], JHEP 1204, 033 (2012) [arXiv:1203.3976 [hep-ex]].

[6] S. Chatrchyan et al. [CMS Collaboration], JINST 3, S08004 (2008).
[7] T. Sjostrand, S. Mrenna and P. Z. Skands, JHEP 0605 , 026 (2006) [hep-ph/0603175].

[8] D. J. Lange, Nucl. Instrum. Meth. A 462, 152 (2001).

[9] K. Nakamura et al. [Particle Data Group Collaboration], J. Phys. G 37, 075021 (2010).

[10] R. Aaij et al. [LHCb Collaboration], Phys. Rev. D 85, 032008 (2012) [arXiv:1111.2357 [hep-ex]].

[11] T. Junk, Nucl. Instrum. Meth. A 434, 435 (1999) [hep-ex/9902006].

[12] A. L. Read, J. Phys. G 28, 2693 (2002).

[13] G. Burdman, E. Golowich, J. L. Hewett and S. Pakvasa, Phys. Rev. D 66, 014009 (2002) [hep$\mathrm{ph} / 0112235]$.

[14] J. C. Anjos et al. [Tagged Photon Spectrometer Collaboration], Phys. Rev. Lett. 62, 1587 (1989). 